\title{
Research and Design of Indoor Environment Monitoring System
}

\author{
Jie Huang \\ College of Mechanical and Electronic Engineering, Hezhou University, Hezhou, China, 542899 \\ huangjie0773@163.com
}

Keywords: Indoor environment; Monitoring system; Zigbee; GSM

\begin{abstract}
In order to monitor and control the indoor harmful gas content and indoor air quality timely and accurately, an indoor environment monitoring system was studied and designed by Zigbee wireless communication technology and GSM technology. The content of formaldehyde, gas and natural gas can be monitored and controlled in real time. In this paper, the design principle of the monitoring node and control node of the indoor environment monitoring system is described. The system has been tested. Test results show that its functions and performance indicators meet the design requirements. It has the advantages of stable performance and convenient operation. It has broad application prospects.
\end{abstract}

\section{Introduction}

In recent years, many tragedies have happened because of formaldehyde poisoning and gas poisoning. With the emergence of fog and haze, indoor air quality has also been more and more people's attention. On the one hand, the indoor environment monitoring system usually uses the wired monitoring method. System wiring is not convenient. Extension of the system is not convenient [1]. On the other hand, the indoor environment monitoring system usually does not have the function of remote monitoring and control. The monitoring data of the system can be viewed only in the room. It is not convenient to use. When the user is not in the indoor, if the indoor gas content is too high or natural gas content is too high, the user can not understand in time. Zigbee is a low cost, low transmission rate, short distance and high reliability of wireless communication technology [2, 3]. Zigbee technology has a very wide range of applications in the smart home and building automation. It has the characteristic of convenient wiring and flexible networking [4, 5]. Combined with Zigbee wireless communication technology and GSM technology, an indoor environment monitoring system based on Zigbee technology and GSM network was studied and designed. The content of formaldehyde, gas and natural gas can be monitored and controlled in real time by system through the Zigbee wireless network and GSM network.

\section{System Structure}

The system consists of monitoring node, control node, coordinator, Web server and monitoring terminal. The structure of the system is shown in Fig. 1. Monitoring node, control node and coordinator set up the network through Zigbee technology. The coordinator and the Web server are connected by serial port. The GSM module and the Web server are connected by serial port. The monitoring terminal can be a PC or a mobile phone. PC and mobile phones can connect and communicate with the Web server through the internet network. Mobile phones can also be connected to the Web server through the GSM network. 


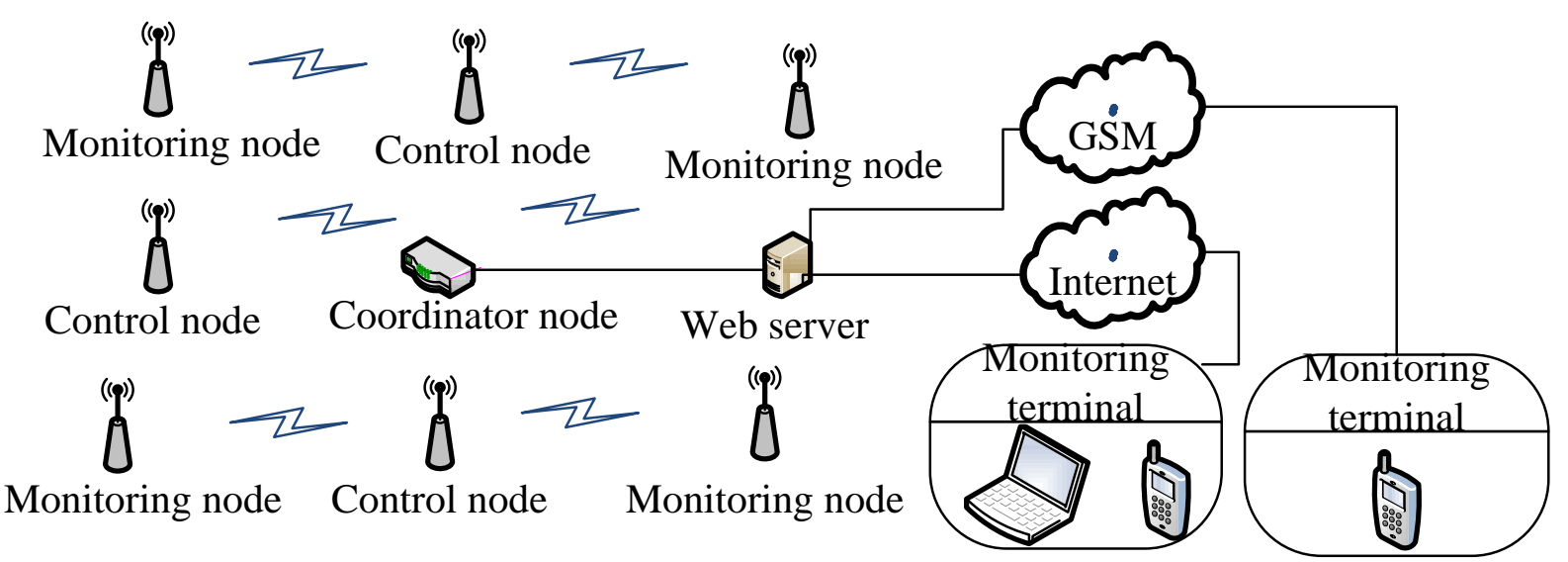

Figure 1. System structure

\section{System Principle and Hardware Design}

Formaldehyde content, gas content, natural gas content and air quality were monitored by monitoring nodes. The monitoring data is transmitted to the coordinator through the Zigbee wireless network. Control node is used for opening and closing the window, the fan and the exhaust fan in the room. Web server receives the monitoring data of each monitoring node through the serial port. Data is analyzed, updated and stored by the Web server. If the gas content is abnormal or the natural gas content is abnormal, it sends out the alarm signal. At the same time, the content of gas or natural gas is regulated by the system through the control of the window or the exhaust fan. On the one hand, users can view the indoor monitoring data and alarm information through the login Web server. Users can also remotely control the windows, fans, and exhaust fans by the monitoring interface. On the other hand, users can also view the indoor monitoring data through mobile phones and GSM network. Users can also control windows, fans and exhaust fans through mobile phones and GSM networks. If the gas content is too high or the natural gas content is too high, the user can receive the alarm information of the system through the mobile phone. According to the alarm information, users can take the appropriate treatment measures in time.

Hardware Design of Monitoring Node. The monitoring node is composed of the formaldehyde content detection module, the gas content detection module, the natural gas content detection module, the air quality detection module, the keyboard, the alarm circuit and the LCD display. The hardware structure of the monitoring node is shown in Fig. 2.

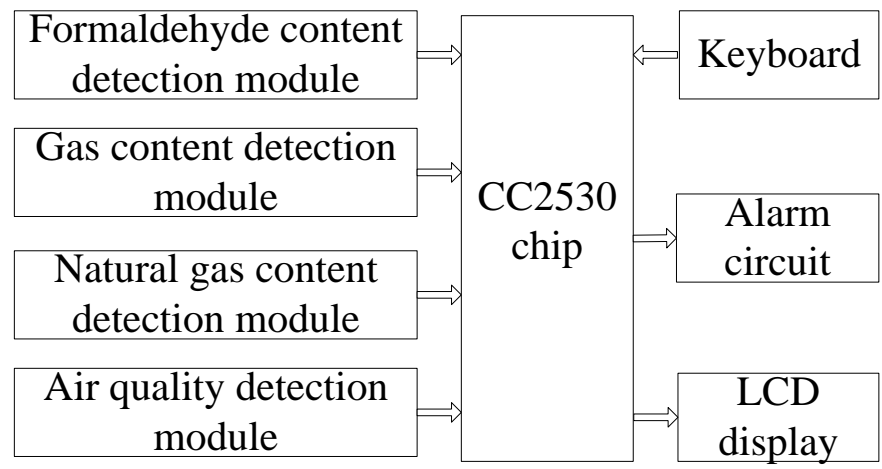

Figure 2. Hardware structure block diagram of monitoring node

Limited to the length of the article, the article only describes the gas content detection circuit. Gas content detection circuit as shown in Fig. 3. 


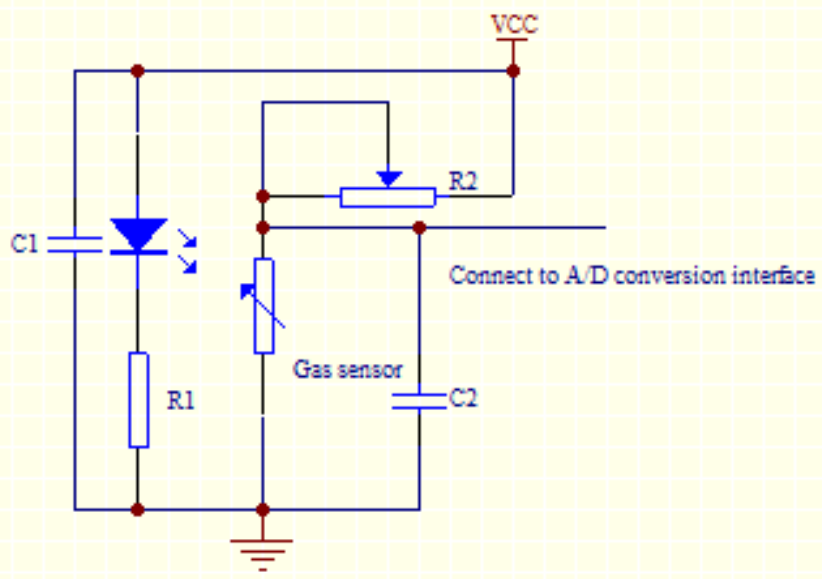

Figure 3. Gas content detection circuit

Hardware Design of Control Node. Control node includes window control node, fan control node and exhaust fan control node. The window control node controls the opening and closing of the window through the window control module. It is composed of a motor drive module, a motor and a stroke switch. It controls the window through the motor and the travel switch. The travel switch is used to detect the location of the window. The fan control node controls the opening and closing of the fan through the fan control module. It includes a plurality of relays. It controls the fan through the relay. The exhaust fan control node controls the opening and closing of the exhaust fan through the exhaust fan control module. The hardware structure of the control node of the exhaust fan is shown in Fig. 4. It controls the exhaust fan through the relay. A photoelectric sensor is arranged on the exhaust fan. System through the photoelectric sensor to detect whether there is a fault in the exhaust fan. It contains a main exhaust fan and a spare exhaust fan. When the main exhaust fan failure or other abnormal situation occurs, a spare exhaust fan starts work.

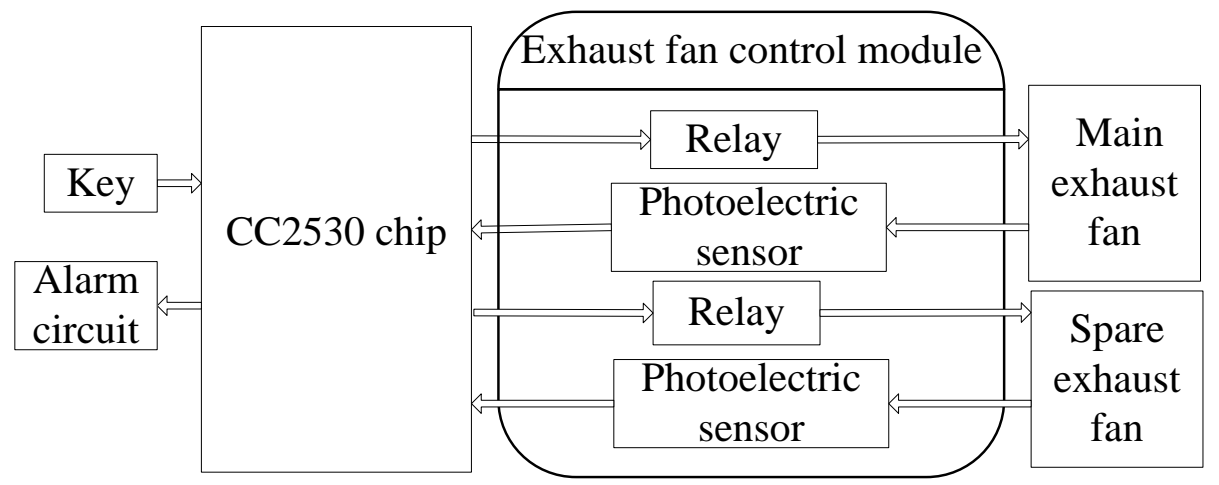

Figure 4. Hardware structure block diagram of exhaust fan

\section{Software Design of System}

Communication Protocol of Monitoring Node. The monitoring data is transmitted to the Web server through the Zigbee network. At the same time, Web server also needs to send query information or control commands to monitoring node through the Zigbee network. Monitoring node and Web server must follow the standard of communication protocol in the transmission of data. The transmission data communication protocol of the monitoring node is shown in Table 1.

The length of the data header is 1 byte. It is a fixed value 0x00. Monitor node's address length is two bytes. Data property indicates the type of data to be transmitted. Data length is the length of the actual data in the data content field. 
Table 1 Data transmission protocol for monitoring node

\begin{tabular}{cccccc}
\hline $\begin{array}{c}\text { Data } \\
\text { header }\end{array}$ & $\begin{array}{c}\text { Monitor node } \\
\text { address }\end{array}$ & $\begin{array}{c}\text { Data } \\
\text { attribute }\end{array}$ & Data length & Data content & Check code \\
\hline $\begin{array}{c}\text { Its length } \\
\text { is one byte }\end{array}$ & $\begin{array}{c}\text { Its length is } \\
\text { two bytes }\end{array}$ & $\begin{array}{c}\text { Its length } \\
\text { is one byte }\end{array}$ & $\begin{array}{c}\text { Its length is } \\
\text { one byte }\end{array}$ & $\begin{array}{c}\text { Its length is } \\
\text { one byte to } \\
\text { eight bytes }\end{array}$ & $\begin{array}{c}\text { Its length is } \\
\text { one byte }\end{array}$ \\
\hline
\end{tabular}

Program Design Flow Chart of the Web Server. Web server receives the monitoring data of each monitoring node through the serial port $[8,9]$. At the same time, it can also send information to monitor node and control node through the serial port. Users can view the monitoring data in real time through the login Web serve. It can also be controlled the windows, the fans and the exhaust fans through the monitoring interface. The program design flow of Web server is shown in Fig. 5.

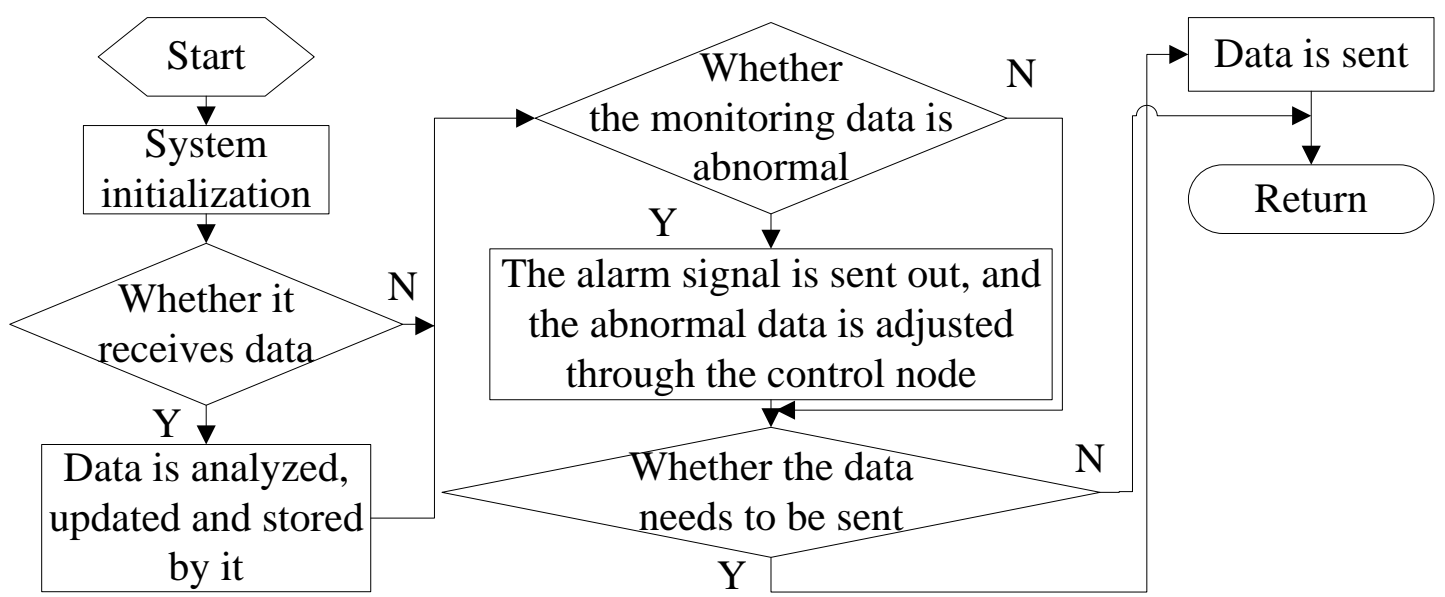

Figure 5. Program design flow chart of the Web server

\section{System Test}

In order to test the function and performance index of the system, it is installed in a set of housing. It has four rooms, a kitchen, two bathrooms and a living room. Eight monitoring nodes, six control nodes, a coordinator and a Web server are installed in the housing. The test data of the system are shown in Table 2. Monitoring data in the Table 2 is the measurement data of the system. The measured data in the Table 2 is measured by the instrument.

Table 2 System test data

\begin{tabular}{ccccccc}
\hline Test item & $\begin{array}{c}\text { Monitoring } \\
\text { data }\end{array}$ & $\begin{array}{c}\text { Measured } \\
\text { data }\end{array}$ & $\begin{array}{c}\text { Relative } \\
\text { error }\end{array}$ & $\begin{array}{c}\text { Alarm } \\
\text { status }\end{array}$ & $\begin{array}{c}\text { Exhaust fan } \\
\text { working status }\end{array}$ & $\begin{array}{c}\text { Window } \\
\text { status }\end{array}$ \\
\hline $\begin{array}{c}\text { Formaldehyde } \\
\text { content } \\
\text { Gas content }\end{array}$ & $0.012[\mathrm{ppm}]$ & $0.0125[\mathrm{ppm}]$ & $4.2 \%$ & Alarm & Run & Open \\
$\begin{array}{c}\text { Natural gas } \\
\text { content }\end{array}$ & $0.0[\mathrm{ppm}]$ & $0.0[\mathrm{ppm}]$ & $0.0 \%$ & $\begin{array}{c}\text { No } \\
\text { alarm }\end{array}$ & Stop & $\begin{array}{c}\text { Keep the } \\
\text { original } \\
\text { state }\end{array}$ \\
$\begin{array}{c}\text { Natural gas } \\
\text { content }\end{array}$ & $20.0[\mathrm{ppm}]$ & $19.9[\mathrm{ppm}]$ & $0.5 \%$ & Alarm & Run & $\begin{array}{c}\text { Keep the } \\
\text { original } \\
\text { state } \\
\text { Open }\end{array}$ \\
\hline
\end{tabular}


Test data show that the system can accurately monitor the content of formaldehyde, gas, natural gas. If the gas content is abnormal or the gas content is abnormal, it sends out the alarm signal. At the same time, the content of coal gas or natural gas is regulated by the system through the control of the window or the exhaust fan. Users can view the monitoring data in real time through the login Web serve. It can also be controlled the windows, the fans and the exhaust fans through the monitoring interface.

\section{Conclusions}

Combined with Zigbee wireless communication technology and GSM technology, an indoor environment monitoring system was studied and designed. It can accurately monitor and control the formaldehyde content, gas content and air quality in the room. Users can remotely view the indoor monitoring data through the PC or mobile phone. It is also capable of remote control of windows, fans and exhaust fans through the PC or mobile phone. It is very convenient to use. When the indoor formaldehyde content is too high or the gas content is too high or some other abnormal situation occurs, the user can receive the alarm information of the system through the mobile phone. According to the alarm information, users can take the appropriate treatment measures in time. It has the advantages of convenient operation and good stability. It has broad application prospects.

\section{Acknowledgement}

This work was financially supported by the National Natural Science Foundation of China (Grant No. 6154055).

\section{References}

[1] H.J. Zhou, Y.L. Zhang, Z. Jin and W.L. Liu: Application of Electronic Technique, Vol. 41 (2015) No.2, p.86. (In Chinese)

[2] H.B. Yu, W. Liang and P. Zeng: Intelligent Wireless Sensor Network System (Science Press, Chinese 2013), P.58. (In Chinese)

[3] X.Q. Wang, Y.J. Ou and N.L. Huang: Design and Implementation of ZigBee Wireless Sensor Network (Chemical Industry Press, Chinese 2012), p.80. (In Chinese)

[4] H.Y. Yang, W.T. Zhang and W.T. Feng: Jiangsu Agriculture Sciences, Vol. 44 (2016) No.9, p.401. (In Chinese)

[5] L.M. Sun, J.Z. Li, Y. Chen and H.S. Zhu: Wireless Sensor Network (Tsinghua University press, Chinese 2005), p.62. (In Chinese)

[6] W.J. Yang, Y.F. Chen and X.D. Wang: Electronic Design Engineering, Vol. 22 (2014) No.18, p.95. (In Chinese)

[7] X.L. Feng, Y.G. Jiang, F. Song and Q. Meng: Measurement \& Control Technology, Vol. 33 (2014) No.8, p.88. (In Chinese)

[8] H. Yu and H.Z. Ma: Industrial Control Computer, Vol. 8 (2015) No.4, p.313. (In Chinese)

[9] W.M. Zhang: Computer Applications and Software, Vol. 32 (2015) No.3, p.73. (In Chinese)

[10] X. Huang: Bulletin of Science and Technology, Vol. 28 (2012) No.8, p.120. (In Chinese) 